
\title{
3 Research Soure \\ The Pitfalls and Strategies of Endoscopic Localization in Laparoscopic Colectomy: A Case Report
}

\section{Wei Wang}

Shaoxing People's Hospital https://orcid.org/0000-0003-3185-2487

\section{Feng Tao}

Shaoxing People's Hospital

Jieqing Lv ( $\sim$ jieqingzg@sina.com )

Shaoxing Hospital, Zhejiang University School of Medicine

\section{Case report}

Keywords: Intraoperative localization, Accuracy, Colonoscopy, Laparoscopic colectomy

Posted Date: April 5th, 2021

DOI: https://doi.org/10.21203/rs.3.rs-375257/v1

License: (c) (1) This work is licensed under a Creative Commons Attribution 4.0 International License.

Read Full License 


\section{Abstract}

Background: laparoscopic segmental colectomy is suitable for removing difficult polyps that are large, broad-based, or located in tortuous bowel segments. As we know, accurate segmental resection depends on precise localization. So far, intraoperative labeling of lesions by colonoscopy is increasingly performed for achieving appropriate resection margins, but a certain deviation is also found. There is no unified and standard endoscopic polyp localization method at present.

Case presentation: A 63-year-old woman was admitted because she was diagnosed as a large and broadbased colonic polyp which was unsuitable for colonoscopic polypectomy. During endoscopy-assisted laparoscopic segmental colectomy, the irradiation angle of colonoscopy light on the polyp head was responsible for the localization errors. We proposed three-step measures of correct endoscopic polyp localization to ensure the accurate resection in laparoscopic segmental colectomy.

Conclusions: Three-step measures of correct endoscopic polyp localization ensured the successful resection of colonic polyps in laparoscopic colectomy. Their advantages include simplicity, practicality and reliable localization.

\section{Background}

Colonic adenomas are common types of colonic polyps and belong to precancerous lesions [1-4]. They should be removed to prevent the transformation of adenoma to adenocarcinoma [4]. Significantly, colonoscopic polypectomy is unsafe for those patients with difficult polyps that are large, broad-based, or located in tortuous bowel segments because of uncontrollable bleeding, perforation and inadequate margins $[5,6]$. While laparoscopic colectomy is more appropriate because of larger resection range, especially for those suspected malignant polyps. However, the most obvious disadvantage of laparoscopic colectomy is the lack of tactility, which leads to the difficulties in defining the precise location of colonic lesions. As we know, accurate segmental resection depends on precise localization. So far, the commonly used positioning methods include preoperative metal clips [7], preoperative dye solutions tattooing $[8]$ and intraoperative endoscopy $[9,10]$.

Preoperative localization of lesions include dye solutions tattooing and metal clips. For the first technology, endoscopists label the bowel segment to be removed with dye solutions, such as methylene blue or sterile India ink [8]. However, several problems have been found. Firstly, preoperative injection of dye solutions directly into the bowel wall may lead to large area spreads of dye solutions in the colonic wall, which result in further difficulties in determining the appropriate resection margin. Secondly, colonic perforation can easily be caused. And extravasation of dye solutions and digestive juice into the abdominal cavity may lead to abdominal infection, adhesions and intestinal obstruction [11]. For the second technology, endoscopists label the bowel segment to be removed with metal clips. They grip onto the targeted colonic mucosa during colonoscopy [7]. Although clip marking is accurate, because metal 
clips are small and located in colonic cavity, they are invisible and impalpable by laparoscopic operators [7], especially for those clips located on the mesenteric side of colon.

Intraoperative localization by colonoscopy is widely used. Because it has advantages such as real-time orientation, easy operation, no pain and so on. Moreover, there is no risk of clip off or dye diffusion. The only disadvantage is flatulence, which can be prevented by blocking the proximal bowel with a laparoscopic lossless forcep at the beginning of colonoscopy and absorbing air adequately at the end of colonoscopy. There are few reported case analyses of colonoscopic labeling failure. In this case, we encountered the embarrassing situation that no lesions were in the surgical specimen because of inappropriate colonoscopy localization. We have made a comprehensive analysis of this case, hoping that laparoscopic surgeons learn from this case and avoid repeating similar mistakes.

\section{Case Presentation}

A 63-year-old woman was admitted because she was diagnosed as colonic adenoma in the local hospital after colonoscopy. She was transferred to our hospital for laparoscopic colectomy because the broadbased colonic polyp was large(Fig. 1A)and not suitable for colonoscopic polypectomy. After the preoperative examinations, this patient received endoscopy-assisted laparoscopic segmental colectomy on October 20, 2020. During operation, the lesion was found by colonoscopy after the establishment of pneumoperitoneum. Then we took the bright spot of colonoscopy as the center of the lesion during laparoscopy. And the bilateral surgical margins were $4 \mathrm{~cm}$ away from the center. This segmental colectomy was performed successfully. Because we believed in the accuracy of endoscopic guidance so much, colonic parallel and cross-to-overlap anastomosis was performed directly without taking out the specimen for positioning check. The specimen was not taken out from the abdominal cavity until the anastomosis was finished. But unfortunately, the polyp was not in the surgical specimen. We had to locate the lesion again. We did a small push action toward the root of polyp using endoscopy top, and looked for the undulating light spot outside the colon under laparoscope. A small push action was performed with a laparoscopic forcep at this light spot outside the colon. We found that the undulating point was the root of polyp with colonoscopy. Then we made a mark using metal clips outside the colon and performed a second segmental colectomy $\mathrm{Fig}$. 1B囚. Because of the initial inaccurate colonoscopic localization, the operation time was prolonged, the medical expenses increased, and the surgeons felt unexpected failure. The postoperative pathology showed tubular adenoma as well as high grade intraepithelial neoplasia[Fig. $1 \mathrm{C} \rrbracket$.

\section{Discussion And Conclusions}

Because of the advantages such as real-time orientation, easy operation, no pain and so on, intraoperative colonoscopy in laparoscopic colectomy is regarded as an efficient lesion positioning approach for selected patients with large or broad-based polyps $[9,10]$. As we know, the accuracy of lesion location is very important, which directly determines the accuracy of colonic resection margin, especially for patients with benign lesions who intend to undergo local bowel segmental resection. By 
precise colonoscopic localization of the lesions, long segment colectomy usually could be avoided. Generally speaking, slight deviations of colonic resection margin can be accepted. Large deviations like this case are relatively fewer.

In this case, we took the bright spot of colonoscopy as the center of the lesion under laparoscopy. And the bilateral surgical margins were $4 \mathrm{~cm}$ away from the center. Therefore, we thought that the lesion should be in the specimen. Then, we anastomosed the colonic stumps firstly and removed the specimen from the abdominal cavity secondly. However, there was no lesion in the specimen. This situation was frustrating. The possible causes were as follows: 1 . The distance between the endoscopic light source and the reflective bowel wall can be long or short, which is uncertain. It mainly depends on the angle of the endoscopic light. 2. Pedicle polyps have certain mobility. Therefore, there is a certain deviation if operators focus on the polyp itself rather than the polyp pedicle. In other words, polyp-pedicle localization is more accurate than polyp-body localization.

After analyzing the different irradiation angles of colonoscopy light on polyps, we believe that there are three situations of maximum localization errors as showed in Fig. 2A. These polyps are situated in either direct colonic segments or tortuous colonic segments. The irradiation angle of colonoscopy light on the polyp head and parallel to the colon is responsible for these localization errors. However, these are the most common situations of colonoscopy, and so is gastroscopy actually. In other words, we need to adjust the angle of the endoscopic light source so that the light scattering is the least, and the localization is more accurate. And necessary verification methods should be carried out to ensure accurate localization.

In order to avoid the recurrence of similar incidents, we try to learn from this mistake. There are some suggestions given to peers for ensuring the accuracy of endoscopy localization in laparoscopic segmental colectomy: 1 . The direction of colonoscopy light should be toward the root of polyp. Operators do a small push action using endoscopy top, and look for the undulating light spot outside the colon with laparoscope. 2. A small push action is performed with a laparoscopic forcep at this light spot outside the colon. Operators observe whether the undulating point is the root of polyp with colonoscopy. If the internal and external localizations are consistent, make a mark such as metal clips outside the colon. 3 . Before colonic anastomosis, the specimen should be taken out immediately after being cut off for localization check(Fig. 2B). These three-step measures provide three-tier insurance. Their advantages include simplicity, practicality and reliable localization. These three-step measures deserve to be popularized not only in colonoscopy assisted operations, but also in gastroscope assisted operations.

\section{Declarations}

Ethics approval and consent to participate The study was approved by our hospital ethics committee in advance. Informed consent was obtained from the patient for participation.

Consent for publication Consent was obtained from the patient for publication. 
Availability of data and material Data and material were available upon request.

Competing interests No conflict of interest to be declared.

Funding No funding was received.

\section{Authors' contributions}

Conceptualization: Wei Wang.

Data curation: Wei Wang.

Methodology: Feng Tao.

Resources: Jieqing LV.

Writing - original draft: Wei Wang.

Writing - review \& editing: Wei Wang, Feng Tao, Jieqing Lv

Acknowledgements We thank this patient for participation and publication.

\section{References}

1. Vogelstein B, Fearon ER, Hamilton SR, et al. Genetic alterations during colorectal-tumor development. N Engl J Med. 1988;319:525-32.

2. He X, Hang D, Wu K, et al. Long-term risk of colorectal cancer after removal of conventional adenomas and serrated polyps. Gastroenterology. 2020;158:852-61.e4.

3. Yu J, Zhang Y, Qian J. Endoscopic submucosal dissection in the treatment of patients with early colorectal carcinoma and precancerous lesions. J Gastrointest Oncol. 2020;11:911-7.

4. Sarah B, Placek JN. Combined Endoscopic Laparoscopic Surgery Procedures for Colorectal Surgery. Clin Colon Rectal Surg. 2017;30:145-50.

5. Isabella Plumptre T, Tolppa, Zaynab AR, Jawad N. Zafar. Donut rush to laparoscopy: postpolypectomy electrocoagulation syndrome and the 'pseudo-donut' sign. BJR Case Rep. 2020;6:20190023.

6. Rachel E, Martin, Mark K, Soliman. Laparoscopic management of snare entrapment during colonoscopic polypectomy. J Surg Case Rep. 2018;2018:rjy101.

7. Nguyen MH, Mori K. Localization of a colonic lesion in the era of laparoscopic colectomy. ANZ J Surg. 2011;81:584-6.

8. Hiroaki Uehara T, Yamazaki A, Iwaya, et al. A rare case of peritoneal deposits with carbon pigmentation after preoperative endoscopic tattooing for sigmoid colon cancer. Int $\mathrm{J}$ Colorectal Dis. 2019;34:355-8. 
9. Liu ZH, Liu JW, Chan FS, Li MK. Joe Km Fan. Intraoperative colonoscopy in laparoscopic colorectal surgery: A review of recent publications. Asian J Endosc Surg. 2020;13:19-24.

10. Erika L, Simmerman, Ray S, King P, Benson Ham 3rd, Vendie H. Hooks 3rd. Feasibility and Safety of Intraoperative Colonoscopy after Segmental Colectomy and Primary Anastomosis. Am Surg. 2018;84:1175-9.

11. Singh S, Arif A, Fox C, Basnyat P. Complication after pre-operative India ink tattooing in a colonic lesion. Dig Surg. 2006;23:303-3.

\section{Figures}
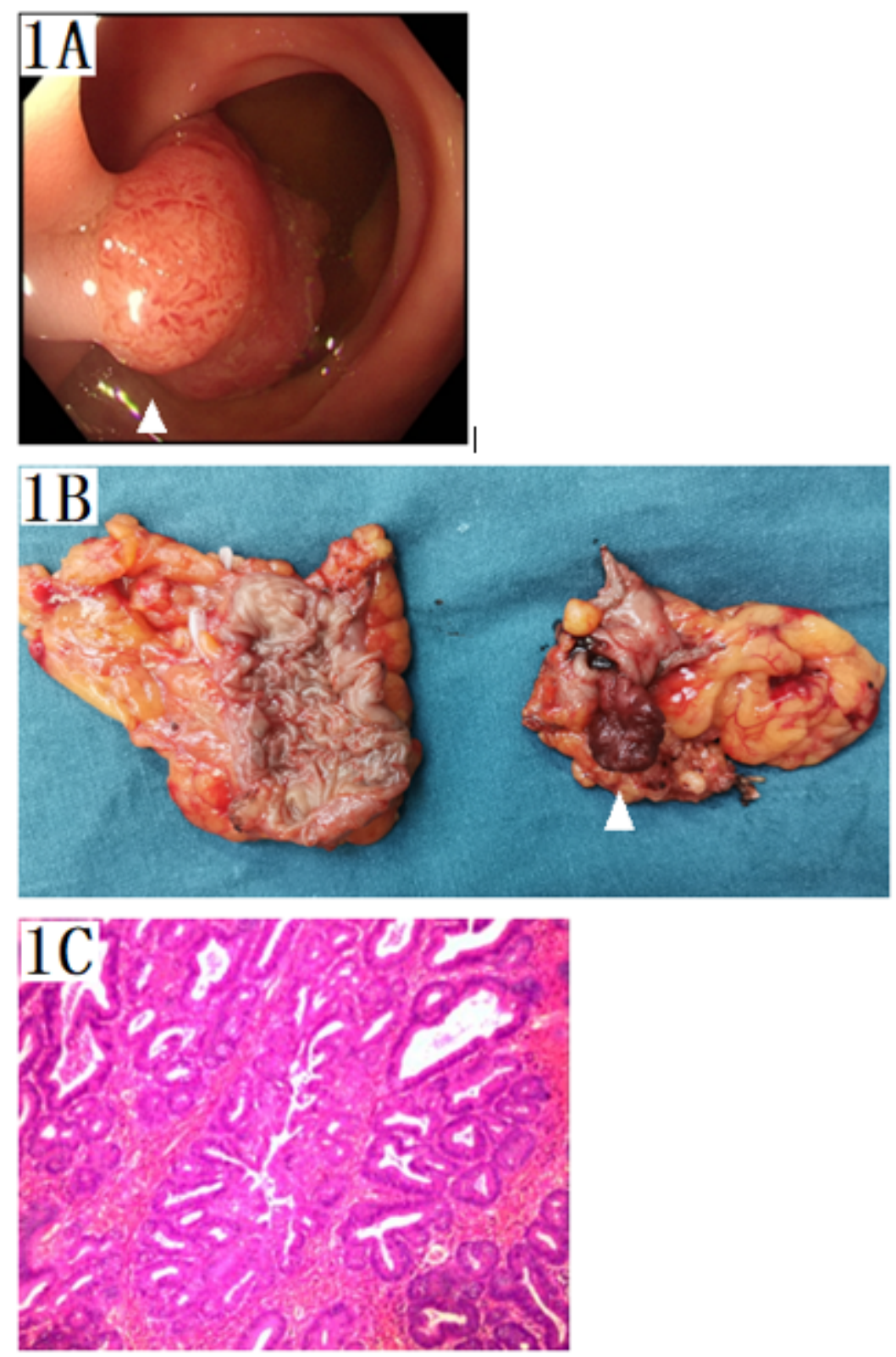

Figure 1 
Photos of the colonic polyp. 1A The broad-based colonic polyp in sigmoid colon was large. 1B The colonic polyp was not in the first surgical specimen on the left. A second segmental colon resection was performed to remove this lesion as showed on the right. 1C The postoperative pathology showed tubular adenoma as well as high grade intraepithelial neoplasia.

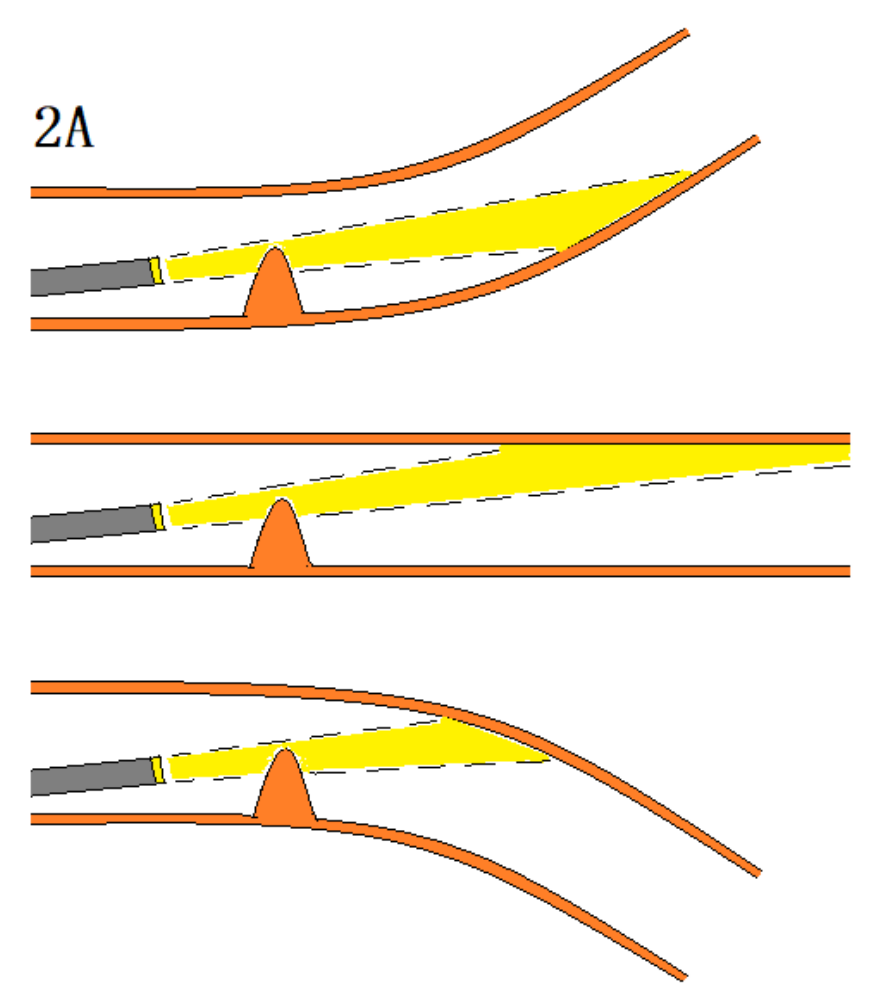

\section{$2 \mathrm{~B}$}
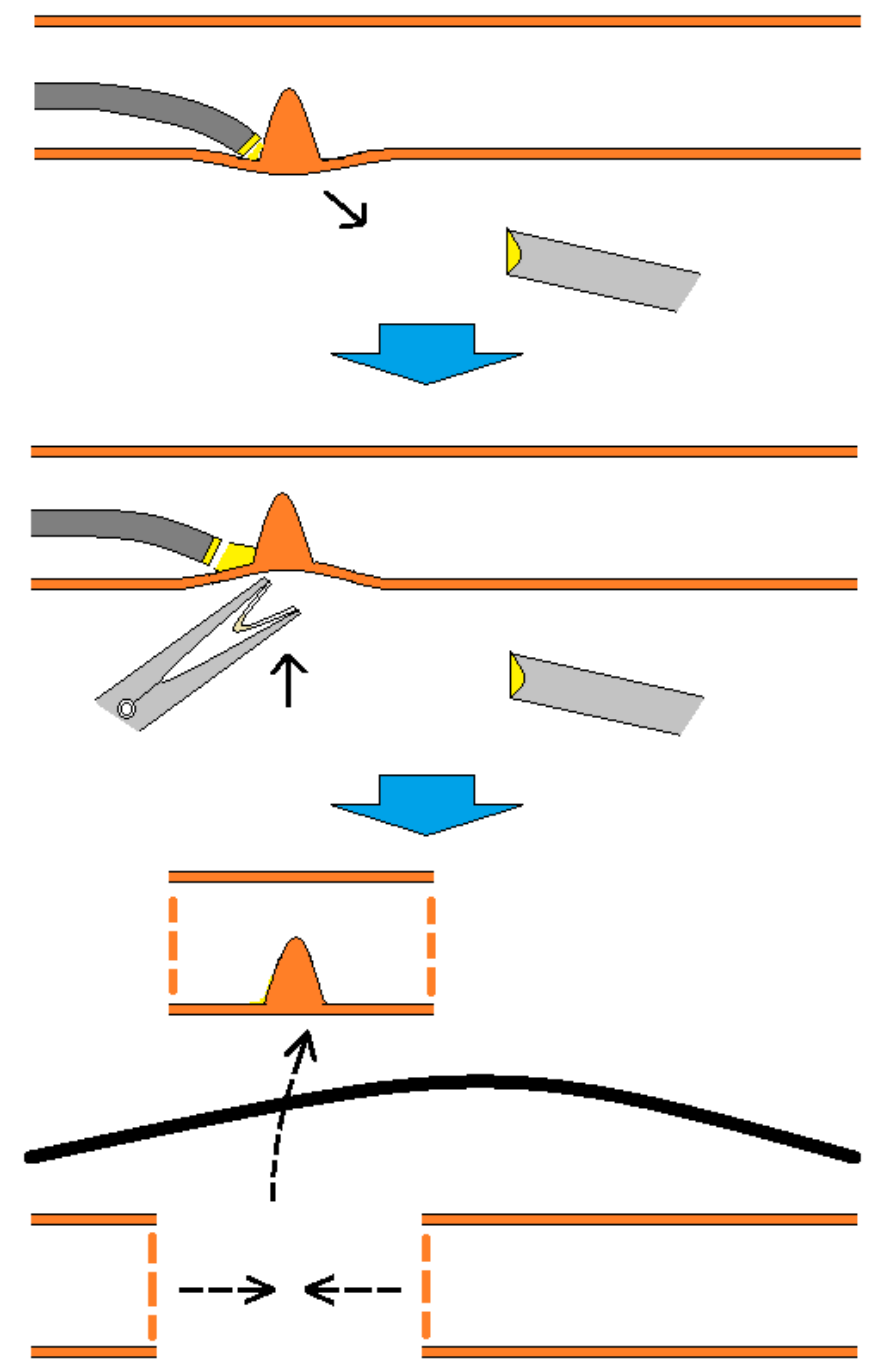

Figure 2

Comparisons between incorrect endoscopic polyp localizations and correct endoscopic polyp localization 2A There are three situations of maximum localization errors, especially for the polyps which are situated in direct colonic segments. 2B Three-step measures of correct endoscopic polyp localization: 1 . The direction of colonoscopy light should be toward the root of polyp. Operators do a small push action using endoscopy top, and look for the undulating light spot outside the colon with laparoscope. 2. A small push action is performed with a laparoscopic forcep at this light spot outside the colon. Operators observe whether the undulating point is the root of polyp with colonoscopy. If the internal and external localizations are consistent, make a mark such as metal clips outside the colon. 3. Before colonic anastomosis, the specimen should be taken out immediately after being cut off for localization check. 